\title{
Prefrontal involvement in imitation learning of hand actions: Effects of practice and expertise
}

\author{
Stefan Vogt, ${ }^{\mathrm{a}, \mathrm{b}, *}$ Giovanni Buccino, ${ }^{\mathrm{c}}$ Afra M. Wohlschläger, ${ }^{\mathrm{d}}$ Nicola Canessa, ${ }^{\mathrm{e}}$ N. Jon Shah, ${ }^{\mathrm{f}}$ \\ Karl Zilles, ${ }^{\text {f,g }}$ Simon B. Eickhoff, ${ }^{f}$ Hans-Joachim Freund, ${ }^{f}$ \\ Giacomo Rizzolatti, ${ }^{\mathrm{c}}$ and Gereon R. Fink ${ }^{\mathrm{f}, \mathrm{h}}$
}

${ }^{a}$ Department of Psychology, Lancaster University, UK

${ }^{\mathrm{b}}$ Magnetic Resonance and Image Analysis Research Centre, University of Liverpool, UK

${ }^{\mathrm{c} D i p a r t i m e n t o ~ d i ~ N e u r o s c i e n z e, ~ U n i v e r s i t a ̀ ~ d i ~ P a r m a, ~ I t a l y ~}$

${ }^{\mathrm{d}}$ Departments of Neurology, Nuclear Medicine, and Neuroradiology, Technical University Munich, Germany

${ }^{\mathrm{e}}$ CRESA, San Raffaele Scientific Institute, Milan, Italy

${ }_{\mathrm{f}}^{\mathrm{f}}$ Institut für Medizin, Forschungszentrum Jülich, Germany

${ }^{\mathrm{g}}$ C. \& O. Vogt-Institut für Hirnforschung, Heinrich-Heine Universität Düsseldorf, Germany

${ }^{\mathrm{h}}$ Klinik und Poliklinik für Neurologie, Uniklinik Köln, Germany

Received 21 February 2007; revised 2 May 2007; accepted 6 July 2007

In this event-related fMRI study, we demonstrate the effects of a single session of practising configural hand actions (guitar chords) on cortical activations during observation, motor preparation and imitative execution. During the observation of non-practised actions, the mirror neuron system (MNS), consisting of inferior parietal and ventral premotor areas, was more strongly activated than for the practised actions. This finding indicates a strong role of the MNS in the early stages of imitation learning. In addition, the left dorsolateral prefrontal cortex (DLPFC) was selectively involved during observation and motor preparation of the non-practised chords. This finding confirms Buccino et al.'s [Buccino, G., Vogt, S., Ritzl, A., Fink, G.R., Zilles, K., Freund, H.-J., Rizzolatti, G., 2004a. Neural circuits underlying imitation learning of hand actions: an event-related fMRI study. Neuron 42, 323-334] model of imitation learning: for actions that are not yet part of the observer's motor repertoire, DLPFC engages in operations of selection and combination of existing, elementary representations in the MNS. The pattern of prefrontal activations further supports Shallice's [Shallice, T., 2004. The fractionation of supervisory control. In: Gazzaniga, M.S. (Ed.), The Cognitive Neurosciences, Third edition. MIT Press, Cambridge, MA, pp. 943956] proposal of a dominant role of the left DLPFC in modulating lower level systems and of a dominant role of the right DLPFC in monitoring operations.

(c) 2007 Elsevier Inc. All rights reserved.

Keywords: Mirror neuron system; Dorsolateral prefrontal cortex; Action observation; Imitation; Expertise; Motor learning

\footnotetext{
* Corresponding author. Department of Psychology, Lancaster University, Lancaster LA1 4YF, UK. Fax: +44 1524593744.

E-mail address: s.vogt@lancaster.ac.uk (S. Vogt).

Available online on ScienceDirect (www.sciencedirect.com).
}

\section{Introduction}

Imitation learning is the set of processes by which a novel observed action is incorporated into the observer's own motor repertoire. Behavioural work on sequence learning (Bird and Heyes, 2005; Vogt, 1995) and force field adaptation (Mattar and Gribble, 2005) indicates that even during pure observational practice, learners engage neurocognitive systems for motor planning and control (for review, see Vogt and Thomaschke, 2007). Using a variety of neurophysiological and neuroimaging methods, the neural mechanisms involved in these processes have been intensely studied over the last 15 years (for review, see Rizzolatti and Craighero, 2004; Iacoboni, 2005). It was shown that action observation indeed evokes activations not only in higher order visual areas but also in areas traditionally known to be devoted to motor functions. The commonly accepted interpretation is that the observed action is directly matched with the observer's own motor prototype of this action (Rizzolatti et al., 2001). In the human brain, two regions have been identified that subserve this perception-action matching: the caudal part of the inferior frontal gyrus including the adjacent ventral premotor cortex and the rostral part of the inferior parietal lobule. This circuit involved in perception-action matching has become known as the "mirror neuron system" (MNS) and is likely to subserve more than a single cognitive function (Rizzolatti, 2005a; Wilson and Knoblich, 2005).

Whereas the early neuroimaging studies on the MNS were addressing perception-action matching during action observation, the study by Iacoboni et al. (1999) demonstrated that the MNS is also involved in movement imitation. However, relatively simple, overlearned actions were used in this initial study and subsequent imitation research (Iacoboni, 2005). Arguably, such acts do not represent the typical situation faced when learning a novel, complex 
action that is not yet in the observer's behavioural repertoire. Thus, until recently, the hypothesis that the MNS also subserves imitation learning (Jeannerod, 1994) remained untested. In order to explore the related neural circuits, Buccino et al. (2004a) conducted a fMRI study where non-guitarists were asked to imitate unfamiliar guitar chords - a task chosen to represent the initial stage of imitation learning. This study yielded two main results. First, the MNS was strongly activated in the imitation condition in all three phases of a trial, action observation, motor preparation and execution. Second, the left dorsolateral prefrontal cortex (DLPFC, most likely area 46) was found activated mainly during motor preparation of imitative execution.

The first finding was unlikely to reflect the well-documented capability of the MNS to represent meaningful actions (Rizzolatti and Craighero, 2004) because the chords were abstract spatial configurations for the non-guitarist participants. More likely, the observed elementary motor acts, in this case the posture of individual fingers located on the fretboard, were represented in the MNS. Thus, the observed motor cortical engagement presumably reflected "low-level resonance" between visual and motor representations rather than "high-level resonance" (Rizzolatti et al., 2002; Rizzolatti, 2005b). In the chord task, the elementary acts (fingers) were simultaneously present in the display, which facilitated the segmentation. Even more complex forms of 'behavioural parsing', specifically in sequential actions, do not need to rely on an understanding of the observed agent's intention but can be conceptualised in more mechanistic terms (Byrne, 2003). The recent study by Borroni et al. (2005) provides an elegant demonstration of low-level resonance when observing cyclic hand movements.

Buccino et al. (2004a) interpreted the involvement of the left DLPFC to indicate the selection and combination of the individual motor elements, as represented in the MNS, into a new motor pattern. Recent behavioural work (Hazeltine et al., in press) has shown that in the learning of configural actions, such as chords on the guitar or piano, indeed individual hand configurations are learned and not just a generalised capacity for visuomotor matching.

We pursued four major aims with the present study: Since the DLPFC had not been previously reported to be involved in imitation learning, our first aim was to replicate the original finding of Buccino et al. (2004a) for unfamiliar chords. Secondly, if their interpretation of the role of left DLPFC is correct, activations in this region should be reduced when the displayed chords are practised. To this end, all participants were given a practice session with four chords one day before the scanning and were tested on imitating the practised as well as four non-practised chords during scanning. Thirdly, we predicted that also activations in the MNS would be reduced for the practised chords, relative to the non-practised chords. Our rationale for this prediction was the expected reduced top-down input from the DLPFC to the MNS, together with reduced attentional demands to identify elementary finger postures and their combination since the practised chords should be available both visually and motorically as complete units (for a review of practicedependent activation changes, see Kelly and Garavan, 2005). ${ }^{1}$

\footnotetext{
${ }^{1}$ Alternatively, it is conceivable that activations in the MNS might be stronger for rehearsed than for novel movements, as recently demonstrated by Cross et al. (2006) during the observation of complex dance sequences. However, their study involved a substantially longer practice period than ours and action observation during fMRI was not followed by execution. Therefore, it is not straightforward to derive predictions from these results for our study.
}

Finally, we also studied a group of expert guitarists, to whom all chords were familiar, using the same experimental protocol. Given the results of previous studies on expertise effects (Calvo-Merino et al., 2005, 2006), we were interested in the level of activation in both the MNS and in prefrontal areas relative to the non-guitarists, and whether the guitarists might exhibit a different pattern of regional activations due to their extensive motor experience and/or the meaningful context (music) that the chord task represented for them.

\section{Materials and methods}

\section{Participants}

Thirty-two healthy, right-handed volunteers (aged 19 to 38 years) participated in the study. The non-guitarists (eight males and eight females, mean age 26.6 years) did not play any musical instrument. Amongst the guitarists (eight males and eight females, mean age 27.3 years), 11 participants were recruited from the Musikhochschule Aachen and had at least 10 years of experience playing the guitar. The other five guitarists were recruited locally and had between three and 10 years of guitar experience. All but one had specialised in classical guitar. Two additional participants were excluded from the analysis due to large head movements $(>5 \mathrm{~mm})$ or undue hand movements in the observation and baseline events. All participants had normal or corrected-to-normal visual acuity. They gave their written informed consent to the experimental procedure, which was approved by the local Ethics Committee.

\section{Design and experimental conditions}

All participants attended a practice session outside the MRI scanner and, one day later, the main experimental session which included the scanning. In the practice session, they learned to imitate a set of four chords (set A or set B) under conditions that resembled the setup in the scanner. In the scanning session, the four practised chords were used together with a set of four non-practised chords. Half of the participants had practised set A and the other half had practised set $\mathrm{B}$. The scanning session was divided into four blocks of 16 trials each. Each block consisted of two subblocks in which each of the eight chords of sets A and B was shown once in quasi-random order. Thus, each chord was shown eight times during the scanning session. In half of the trials, participants physically executed the presented chord (imitation condition, IMI) on a scanner-suitable guitar neck, and in the other half they imagined executing the chord without overt movement (motor imagery condition, MIM). IMI and MIM trials were presented in quasi-random order, and they were indicated by a small green or red square that was presented centrally for $2 \mathrm{~s}$ before the start of each trial. The results of the MIM condition are not reported in the present paper. Thus, the effective design consisted of the factors chord type (non-practised vs. practised chords) and group (non-guitarists vs. guitarists).

Each trial consisted of four events. In Event 1 (observation), participants were required to carefully observe a video clip showing the right hand of a guitarist that repeatedly performed the same guitar chord. In Event 2 (preparation), a blank blue screen was shown, and participants were instructed to visualise the finger positions of the chord and to map these to the fingers of their left hand. This mapping was facilitated by the mirror-symmetric arrangement of the video clips (Koski et al., 2003). Throughout 
Events 1, 2 and 4, the participants' fingers rested on the fretboard in a standard rest position. During the last second of Event 2, a cross of the same colour as the square cue was shown in the center of the screen to remind participants of the task and to indicate that Event 3 was about to start. In Event 3 (imitative execution or motor imagery), the screen colour changed to a darker blue that matched the background of Event 1, and participants either imitated the previously seen chord in a rhythmical manner with their left hand, or they engaged in motor imagery of the same action while their hand remained in the rest position. At the end of imitative execution, participants returned their fingers to the rest position. In Event 4 (rest), the same background as in Event 2 was shown. Participants returned their fingers to the rest position if they had not already done so and disengaged from the task while keeping their hand still.

In order to be able to analyse the three main events (Events 1, 2 and 3) of each condition separately, an event-related design was used. Events 1, 2 and 4 were presented in different ("jittered") durations across trials (Events 1 and $2=4$ to $10 \mathrm{~s}$, Event $3=7 \mathrm{~s}$; Event $4=6$ to $16 \mathrm{~s}$ ), and conditions were presented in quasirandom order. The task and presentation of events in the imitation trials was thus identical to condition IMI in Buccino et al. (2004a), except that chords could be practised or non-practised, and that the average duration of Event 2 now matched that of Events 1 and 3 to allow for contrasts between events.

\section{Apparatus and stimuli}

The same apparatus was used as described in Buccino et al. (2004a), including a short wooden guitar neck without strings (height $20 \mathrm{~cm}$ ), a Sony PX20 data projector and Presentation software (Neurobehavioral Systems, Inc.) to display the visual stimuli. A subset of the video clips from the previous study was used. Set A and set B each consisted of one chord involving two fingers and three chords involving three fingers. Participants did not see their hand during scanning. For later scoring, each participant's hand was videotaped during the scanning session, synchronous with an image of the displayed stimuli. This served the scoring of imitation quality and the elimination of individual events or trials where participants did not follow instructions (i.e., any overt movement during Events 1, 2, or 4; no execution during event 3 ). As a result, $7.6 \%$ of all events were eliminated in the nonguitarist group and $7.3 \%$ in the guitarists. Since Event 4 was used as baseline for most contrasts, particular attention was paid to any finger movement during this event, which accounted for $61 \%$ of all excluded events. In addition, the last $400 \mathrm{~ms}$ of Event 3 and the first $2 \mathrm{~s}$ of Event 4 were principally removed from the analysis since in this interval participants repositioned their hand on the fretboard.

For the practice session, the scanner setup was replicated in a separate room. Participants were lying on a bed, and stimuli were presented on an Eizo 15-in. flat panel display that was mounted approximately $60 \mathrm{~cm}$ above the participant's head. The model stimuli could be swapped against a life image of the participant's hand, providing visual feedback that was used in a small subset of the practice trials.

\section{Instruction and procedure}

At the beginning of the practice session, which was approximately $90 \mathrm{~min}$ long, participants were introduced to the guitar fretboard to the hand's rest position, and to the observation, preparation and execution events of a trial. This was followed by two practice phases of 72 imitation trials each with the four chords of either set A or set B. The practice trials included trials with immediate visual feedback of the hand during execution (1/6 of trials), trials with delayed visual feedback (4 s after the onset of execution; $1 / 6$ of trials) and trials without visual feedback as in the scanner (4/6 of trials). Participants were instructed to observe the model chords attentively and to reproduce them with maximal accuracy and in the same rhythmical manner as shown in the video clips.

The main experimental session commenced with a further practice phase of 24 imitation trials, followed by another 24 trials in which participants learned to engage in motor imagery instead of overt execution of the chords. Then, a full block of 16 trials was run which exactly resembled the procedure in the four scanning blocks. These procedures lasted about $30 \mathrm{~min}$ and were followed by the main scanning session.

In order to assess practice effects behaviourally throughout the experiment, speeded performance tests were run at four time points. Participants performed the four practised chords as fast as possible over a period of $1 \mathrm{~min}$ in a depicted sequence that was different in each test. The first was run half way through the first practice phase, the second at the end of the second practice phase, the third directly before scanning and the last in a posttest after the scanning.

\section{Functional magnetic resonance imaging}

Functional MR data were acquired with a 1.5-T Siemens Sonata whole-body scanner with echoplanar imaging (EPI) capability using the standard radio-frequency head coil. Multislice $\mathrm{T} 2 *$-weighted echo-planar images were obtained from a gradientecho sequence with the following parameters: echo time $\mathrm{TE}=$ $66 \mathrm{~ms}$, repetition time $\mathrm{TR}=3 \mathrm{~s}$, flip angle $=90^{\circ}$, field of view FOV $=200 \mathrm{~mm}$, slice thickness $=4 \mathrm{~mm}$, inter-slice gap $=0.4 \mathrm{~mm}$, in-plane resolution $=3.125 \times 3.125 \mathrm{~mm}^{2}$. The 29 slices covered the whole brain from the cerebellum through to the vertex. For Events 1, 2 and 3, $n=37$ EPI-volumes were acquired per condition and participant and for Event 4, $n=53$ EPI-volumes were acquired.

\section{Image processing, statistical analysis and anatomical localisation}

The entire data analysis was performed with SPM2 (Statistical Parametric Mapping software; The Wellcome Department of Imaging Neuroscience, London, UK; http://www.fil.ion.ucl.ac.uk) running on MATLAB (The Mathworks, Inc., Natick, MA). The first four images of each run were discarded to allow for T1 equilibration effects. For each participant, all volumes were spatially realigned to the first volume of the first session and unwarped to correct for between-scan motion, and a mean image from the realigned volumes was created. This image was spatially normalised to the Montreal Neurological Institute (MNI) EPI brain template available in SPM2. The thereby derived spatial transformation was then applied to the realigned $\mathrm{T} 2 *$ weighted volumes, which after normalisation were resampled in $2 \times 2 \times 2 \mathrm{~mm}^{3}$ voxels using sinc interpolation in space. All functional volumes were then spatially smoothed with a $10-\mathrm{mm}$ full-width half-maximum isotropic Gaussian kernel for the group analysis. 
Data were analysed using a random-effects model (Friston et al., 1999), implemented in a two-level procedure. In the first level, single-subject fMRI responses were modeled in a general linear model (GLM) by a design matrix comprising the onsets and durations of each event according to the experimental condition for each session (Events 1, 2 and 3 of the four conditions IMI/np, $\mathrm{IMI} / \mathrm{pr}, \mathrm{MIM} / \mathrm{np}$ and MIM/pr). The 12 regressors modeling these events were convolved with the canonical hemodynamic response function. Six additional regressors were included into the GLM to account for voxel intensity variations due to absolute head movements, and one further regressor was included to model incorrect events which had to be excluded from their proper task regressors. Parameter estimates for all regressors were obtained by maximum-likelihood estimation. In the second level, corresponding contrast images of the first stage for each participant were entered into one-sample $t$-tests to compute statistical maps for each event of each experimental condition ('basic contrasts'). In addition, direct contrasts between events ( 1 vs. 2 and 2 vs. 3 ) and between chord types (practised vs. non-practised) were computed per participant and were also entered into one-sample $t$-tests on the second level. All between-groups comparisons were performed by means of two-sample $t$-tests. The results reported are significant at $p_{\mathrm{u}}<0.001$ (uncorrected for multiple comparisons). For anatomical localisation, the functional data were referenced to probabilistic cytoarchitectonic maps (Eickhoff et al., 2005).

\section{Results}

\section{Behavioural data}

Practice effects were assessed behaviourally in two ways, via speeded performance tests and via a rating of the accuracy of imitation performance during scanning. Fig. 1 shows the results for speeded performance. Both groups exhibited pronounced practice effects across the four tests $(F=52.92, p<0.001)$, and the guitarists performed this task substantially faster than the non-guitarists $(F=50.28, p<0.001)$. In addition, guitarists showed stronger improvements with practice than non-guitarists, as indicated by a significant interaction term in the related $4 \times 2$ ANOVA $(F=3.33$, $p<0.05$; linear trend $F=6.43$ ). In the posttest, where performance of practised (pr-) and non-practised (np-) chords was directly compared, both groups performed the np-chords significantly slower than the pr-chords, as indicated by a separate $2 \times 2$ ANOVA $(F=59.09, p<0.001)$. This difference was more pronounced in the guitarists, as confirmed by a significant interaction between group and chord type $(F=6.35, p<0.05)$.

The rating of imitation quality was based on transcripts of the finger positions in tablature format, which had been extracted from the video recordings. On a scale of 0 to 8 , the non-guitarists achieved a mean score of 5.3 for the non-practised chords and of 5.8 for the practised chords, and the guitarists achieved scores of 6.0 and 6.6 , respectively. The corresponding $2 \times 2$ ANOVA indicated a significant effect of group $(F=11.09, p<0.01)$. In addition, the practised chords were performed more accurately than the non-practised chords $(F=14.66, p<0.001)$. The latter finding, as well as the speeded performance data after the scanning, confirms that the effects of the practice session were, to a large extent, item specific. Further, the pronounced group differences, particularly in speeded performance, attest the expertise of our

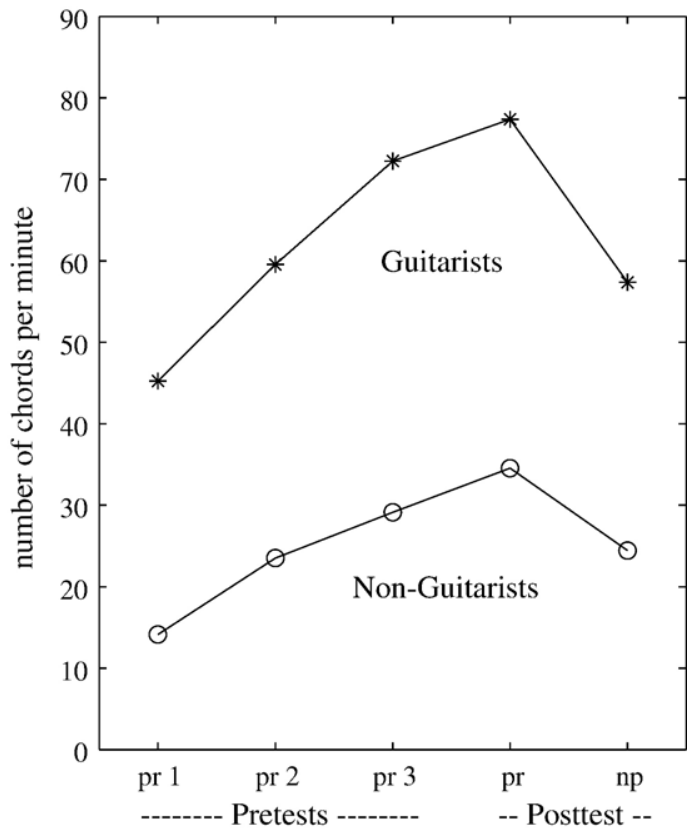

Fig. 1. Speeded performance tests for the practised chords at three time points before scanning (pr 1, 2 and 3), and for practised (pr) and nonpractised (np) chords directly after the scanning. Results indicate clear practice effects in both groups, and a substantially faster overall performance of the guitarists.

guitarist participants. Thus, two crucial preconditions of the study were clearly met.

\section{Functional data}

\section{Common and differential activations between events}

The activations common in action observation, preparation and execution are shown in Fig. 2 (top panel) and Table 1 (overall conjunction between Events 1 to 3 across all participants and both chord types). All three events induced strong activations in the rostral sector of the inferior parietal lobule bilaterally, which extended to left BA2 and right human intraparietal area 2 ('hIP2', see Choi et al., 2006; Eickhoff et al., 2006a,b). In addition, small foci of increased neural activity were found in the superior parietal lobule and in the right posterior inferior temporal gyrus. In the premotor cortex, two distinct foci were present bilaterally. The first was located in the ventral part of the precentral gyrus, extending to the dorsalmost part of the pars opercularis of the inferior frontal gyrus. The second focus (labelled 'PMd' in Fig. 2) was located in the caudalmost part of the superior frontal sulcus bilaterally and extended dorsally to the border between the superior frontal gyrus and the precentral gyrus. Furthermore, the supplementary motor area (SMA) was found activated across events, as well as the left insula, the caudal part of the middle frontal gyrus and the cerebellum (subcortical activations are not considered further in the present paper).

The major increases in activation between events are shown in the bottom panel of Fig. 2 in the form of two superimposed direct contrasts. From Event 1 to Event 2, activation increases were present in the primary sensory-motor areas bilaterally, the parietal operculum, the insula, the SMA and in prefrontal areas. From Event 2 to Event 3, further increases can be seen in the right 


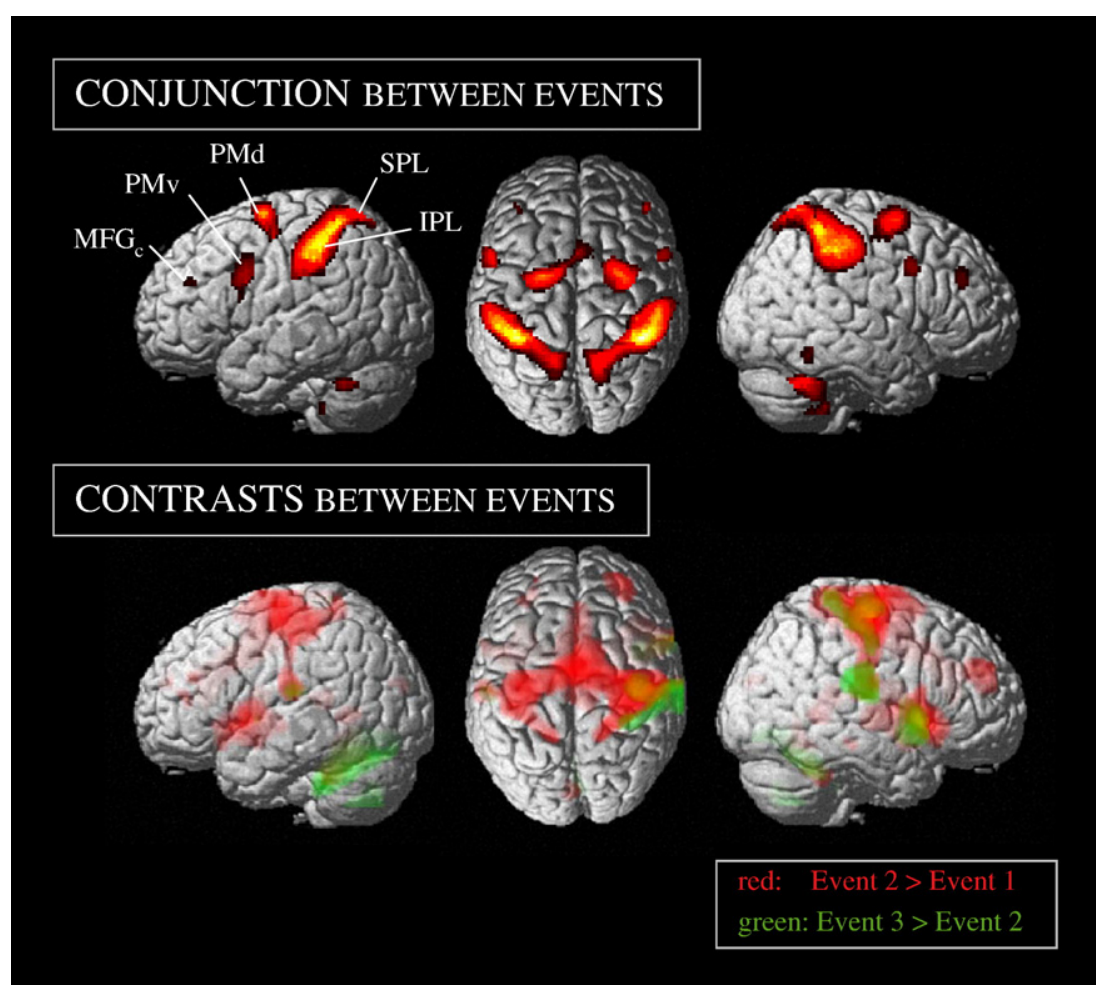

Fig. 2. Top panel: Conjunction between observation, motor preparation and execution (Events 1, 2 and 3 ) across all participants and chord types, indicating common activations across these events. These include the two main regions of the human 'mirror neuron system' (ventral premotor cortex: PMv, and inferior parietal lobule: IPL), as well as the dorsal premotor cortex (PMd), superior parietal lobule (SPL) and the caudal middle frontal gyrus (MFGc). Bottom panel: Direct contrasts indicating increased activations from Event 1 to Event 2 (red blobs) and from Event 2 to Event 3 (green blobs; yellow blobs indicate regions of overlap) across all participants for the non-practised chords (the corresponding contrasts for the practised chords were highly similar). Areas involved in tactile exploration show increased activation from motor preparation to execution.

postcentral gyrus, the right primary motor hand area, the parietal operculum and the insula. ${ }^{2}$ In sum, the results shown in Fig. 2 indicate that the main regions of the MNS, the ventral premotor cortex and the inferior parietal lobule, were consistently involved in observation, preparation and execution, whereas the major activation changes between events were found in the SMA, in primary sensory-motor areas and in further areas involved in tactile exploration (Binkofski et al., 1999; Stoeckel et al., 2003).

\section{Basic contrasts}

We now turn to the individual basic contrasts for each group, chord type and event against Event 4 (baseline) as shown in Fig. 3. The main characteristics of each condition will be described as modulations of the core activation pattern indicated by the conjunction analysis above. Increased activations across events are only reported for areas where the related direct contrast was significant. The main effects of chord type and group and the modulations in prefrontal regions are described in subsequent sections.

As expected, the activations in the non-guitarists for the nonpractised chords (NG/np, see Fig. 3, top left panel) closely resembled those in the corresponding IMI-condition in Buccino et al. (2004a) for each of the three events: During action observation

\footnotetext{
${ }^{2}$ Decreases of activation from Event 1 to Event 2, not shown in Fig. 2, were mainly found in visual areas. From Event 2 to Event 3, widespread decreases were present across the frontal lobe, as well as in posterior parietal areas and the middle and inferior temporal gyrus.
}

(NG/np-1), occipital and posterior temporal regions were found activated in addition to the core pattern of posterior parietal and premotor activations in the conjunction analysis. During motor preparation (NG/np-2), the parieto-premotor circuits remained fully activated despite the absence of further visual input. The inferior parietal activations now extended further ventrally to the left parietal operculum $\left(-56,-18,20^{Z=4.28}\right)$, ' $\mathrm{OP} 1$ ' according to Eickhoff et al. (2006a,b), and they also extended rostrally to area BA2 $\left(-44,-30,48^{Z=6.57} ; 40,-32,42^{Z=5.61}\right)$. The activations in ventral precentral gyrus $\left(-58,6,32^{Z=6.37} ; 60,8,30^{Z=3.97}\right)$ extended to the ventral part of pars opercularis of inferior frontal gyrus (vPO; $52,10,10^{Z=4.25}$ ), most likely BA44. The dorsal premotor activations now extended caudally into precentral gyrus $(-32$, $-16,58^{Z=6.01}$ and $\left.-30,-10,60^{Z=5.94} ; 28,-18,64^{Z=5.93}\right)$. In addition, the mesial wall $\left(-4,-4,62^{Z=6.16}\right)$ and insula $(-32,20$, $2^{Z=4.89}$ ) were now strongly activated. Finally, a further activation focus was present over the right primary motor cortex $(36,-22$, $56^{Z=6.90}$ ) in Event 2, which represents the only discrepancy to our earlier study. During action execution (NG/np-3), the parietal and premotor activations remained highly similar to those in Event 2, with further activation increases in the parietal operculum OP 1 $\left(-54,-22,24^{Z=5.32} ; 56,-18,14^{Z=5.00}\right)$, insula $\left(-40,6,-2^{Z=5.66}\right.$; $42,4,-10^{Z=6.01}$ and $\left.42,6,-2^{Z=5.72}\right)$, right postcentral gyrus (36, $\left.-36,70^{Z=4.99}\right)$ and right primary motor cortex $\left(40,-22,56^{Z=6.77}\right)$.

The activations of the guitarists closely resembled those of the non-guitarists (Fig. 3). The following differences and similarities are worth reporting: When the guitarists observed the non-practised chords (G/np-1), the activation in the left ventral precentral gyrus 
Table 1

Conjunction between Events 1, 2 and 3: Local maxima of activated areas as shown in Fig. 2 (top panel), given in MNI standard brain coordinates [ATB: most probable anatomical region in the Anatomy Toolbox 1.4, Eickhoff et al., 2005; asterisks (*) denote assigned areas]

\begin{tabular}{|c|c|c|c|c|c|c|c|c|c|c|}
\hline \multirow[t]{2}{*}{ Anatomical region } & \multicolumn{5}{|l|}{ Left } & \multicolumn{5}{|c|}{ Right } \\
\hline & $x$ & $y$ & $z$ & $Z$-score & ATB & $x$ & $y$ & $z$ & $Z$-score & ATB \\
\hline Inferior parietal lobule & -42 & -38 & 44 & inf. & $20 \%$ BA 2 & 40 & -40 & 42 & 7.82 & \\
\hline Postcentral gyrus & -42 & -44 & 56 & inf. & $40 \%$ BA $2 *$ & & & & & \\
\hline Human intraparietal area 2 & & & & & & 40 & -44 & 52 & 7.68 & $20 \%$ hIP2 \\
\hline Superior parietal lobule & -14 & -60 & 66 & 6.04 & & 16 & -60 & 64 & 5.90 & \\
\hline Inferior temporal gyrus & & & & & & 62 & -52 & -16 & 3.97 & \\
\hline Precentral gyrus (PMv) & -54 & 6 & 36 & 4.66 & $30 \%$ BA6 & 56 & 10 & 36 & 3.67 & $20 \%$ BA6 \\
\hline Superior frontal gyrus / precentral & -22 & -4 & 68 & 6.59 & $60 \%$ BA6* & 26 & -4 & 56 & 5.59 & $20 \%$ BA6 \\
\hline gyrus (PMd) & -24 & -8 & 56 & 5.88 & $30 \%$ BA 6 & & & & & \\
\hline Supplementary motor area & -2 & 10 & 52 & 4.35 & $70 \%$ BA $6 *$ & & & & & \\
\hline Middle frontal gyrus (caudal) & -38 & 38 & 28 & 3.65 & & 46 & 40 & 28 & 3.79 & \\
\hline Insula & -30 & 20 & 2 & 3.35 & & & & & & \\
\hline Cerebellum (Crus I) & -44 & -52 & -34 & 3.88 & & 40 & -52 & -32 & 5.27 & \\
\hline Cerebellum (Crus II) & -4 & -80 & -38 & 4.52 & & & & & & \\
\hline Cerebellum (VI) & -28 & -62 & -30 & 3.78 & & 30 & -62 & -32 & 5.03 & \\
\hline
\end{tabular}

$\left(-52,8,46^{Z=3.68}\right)$ extended directly to dorsal and ventral parts of the pars opercularis of inferior frontal gyrus (dPO: $-62,12$, $24^{Z=4.36} ; \mathrm{vPO}:-52,12,12^{Z=4.03}$ ), both assigned to BA44 (Eickhoff et al., 2005). In Event G/np-2, the activations in the non-guitarists were again closely matched by the guitarists, including the extension of inferior parietal activations into the parietal operculum OP $1\left(-54,-20,26^{Z=5.41}\right)$ and postcentral gyrus $(-42,-34$, $\left.56^{Z=6.79} ; 36,-34,48^{Z=6.26}\right)$, the activations in ventral precentral gyrus $\left(-54,6,42^{Z=4.81} ; 54,8,40^{Z=4.96}\right)$ and increases in inferior frontal gyrus (dPO: $-56,8,28^{Z=5.09} ; 56,12,30^{Z=4.70}$ and vPO: 56 , $\left.10,10^{Z=5.65}\right)$, the caudal-wards shift of the dorsal premotor activations $\left(-28,-12,60^{Z=6.73} ; 32,-16,58^{Z=6.67}\right)$, the increased activations in the mesial wall $\left(10,-4,62^{Z=6.67}\right)$ and insula $(-44,4$, $10^{Z=5.95}$ ), as well as the strong activation in the right primary sensory-motor areas $\left(44,-22,60^{Z=7.13}\right)$. The latter, unexpected finding did not primarily reflect motor preparatory processes triggered by the cue $1 \mathrm{~s}$ prior to execution, as a reanalysis over the first $5 \mathrm{~s}$ of Event 2 revealed virtually the same activation pattern. During motor execution, the guitarists again showed highly similar activations to the non-guitarists. It is noteworthy that in all four conditions of Event 3, both the ventral part of PO of inferior frontal gyrus $\left(-56,8,8^{Z=\text { inf. }} ; 58,10,8^{Z=\text { inf }}\right)$ and the insula $\left(-40,2,2^{Z=\text { inf; }}\right.$; $44,4,6^{Z=\text { inf }}$ ) exhibited robust activations (coordinates for the main effect of Event 3 across groups and chord types).

A further, only partial commonality across groups was the bilateral activation of the dorsalmost sector of the hippocampus during observation of the practised chords (NG/pr-1: $-24,-30$, $2^{Z=4.28}$ and $-16,-28-8^{Z=3.83} ; 24,-30,2^{Z=3.98} ; \mathrm{G} / \mathrm{pr}-1:-24$, $\left.-32,0^{Z=4.33} ; 28,-30,-2^{Z=4.36}\right)$. During observation of the nonpractised chords, only the guitarists showed a related hippocampal activation $\left(\mathrm{G} / \mathrm{np}-1:-24,-32,0^{Z=4.79} ; 28,-32,0^{Z=4.31}\right)$. This likely reflected that the non-practised chords were familiar to the guitarists only.

\section{Main effects of chord type and group}

Fig. 4 and Table 2 show the direct contrasts between nonpractised and practised chords (np $>$ pr) across groups and separately for each event. They represent the main results of this study. During action observation, stronger activations for the nonpractised chords were present in the superior and inferior parietal lobules, right posterior inferior temporal gyrus, left ventral premotor cortex, caudal superior frontal sulcus and, importantly, the rostral part of left middle frontal gyrus. ${ }^{3}$ During motor preparation (Event 2), the intensity and the extent of differential activations were smaller, and these were confined to the precuneus, inferior parietal lobule approaching the postcentral sulcus and, importantly, two sites in the middle frontal gyrus. Finally, during motor execution, cortical activation differences between chord types were only present in the inferior parietal lobule bilaterally.

Compared to the main effect of chord type, the group differences were distinctly small. During action observation, the main effect of group indicated stronger activations for the guitarists in the right precentral gyrus $\left(14,-24,66^{Z=3.94}\right)$, as well as small foci in the supplementary motor area, the right posterior middle temporal gyrus and inferior temporal gyrus. The non-guitarists showed stronger activations only in the right superior parietal lobule $\left(18,-70,56^{Z=3.68}\right)$. During motor preparation, the guitarists exhibited stronger activations in left inferior parietal lobule $(-56$, $-46,50^{Z=3.75}$ ), right precentral gyrus $\left(48,4,52^{Z=3.55}\right)$, superior frontal gyrus $\left(24,22,62^{Z=3.27}\right)$ and right anterior inferotemporal gyrus $\left(62,-14,-28^{Z=3.31}\right)$. During motor execution, the guitarists showed stronger activations in the left inferior parietal lobule $(-56$, $\left.-42,46^{Z=3.75}\right)$ and in the right parietal operculum OP $4(62,-6$, $8^{Z=3.30}$ ). Conversely, the non-guitarists did not show any enhanced activations during Events 2 and 3.

Only a small set of regions was activated in the interaction contrasts between group and chord type. These regions were either not activated in the related basic contrasts (e.g., the left anterior inferior temporal gyrus in Events 1 and 2; left rostral middle frontal gyrus in Event 3), or they were not of primary interest in the present context (e.g., extrastriate occipital cortex, right precentral gyrus and SMA in Event 2).

\footnotetext{
${ }^{3}$ The inverse main effect of chord type (pr $>n p$ ) indicated extended differential activations in mesial frontal areas, with local maxima in superior frontal gyrus (medial and medial orbital parts) and anterior cingulate gyrus. Also small sectors of the left angular gyrus, right posterior middle temporal gyrus and anterior middle and inferior temporal gyrus were more strongly activated for the practised chords. The mesial frontal and anterior temporal activations were also present in Events 2 and 3. However, none of these activation differences corresponded to positive activations in the related basic contrasts.
} 


\section{OBSERVATION (Event 1)}
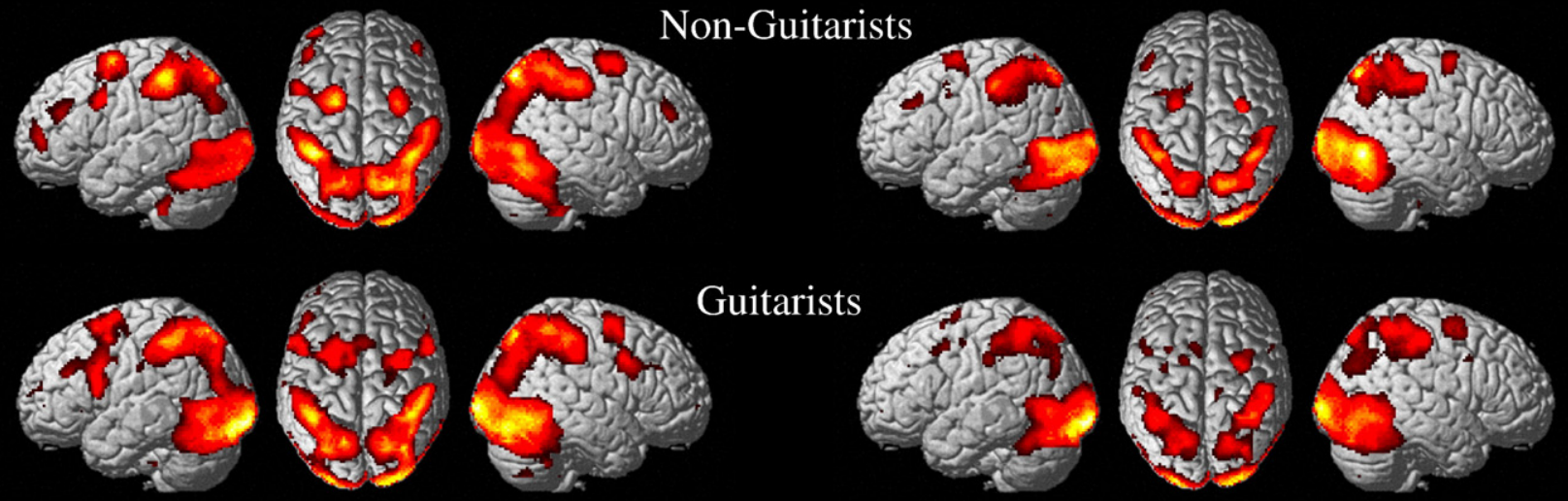

\section{PREPARATION (Event 2)}
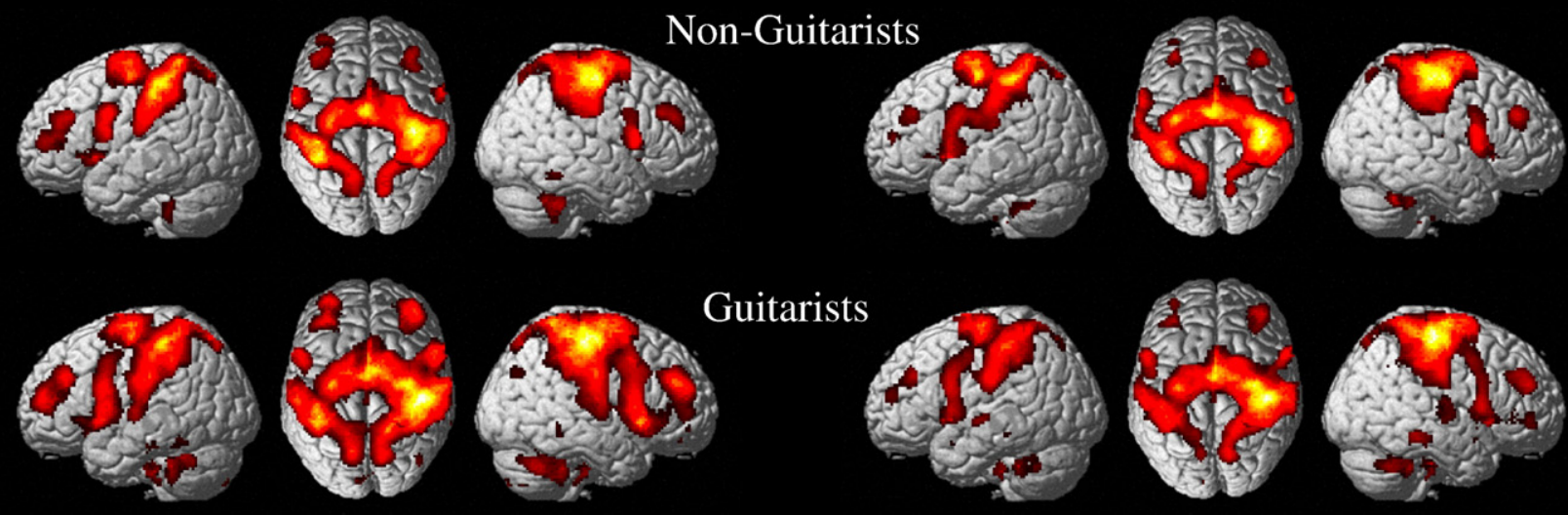

\section{EXECUTION (Event 3)}
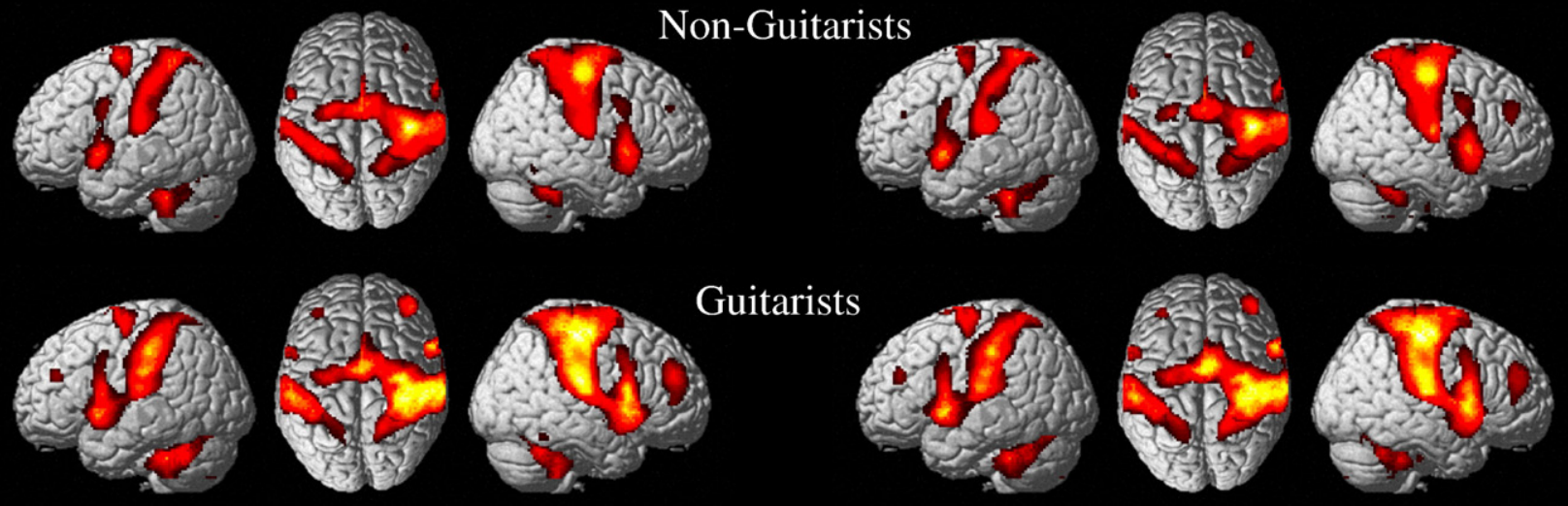


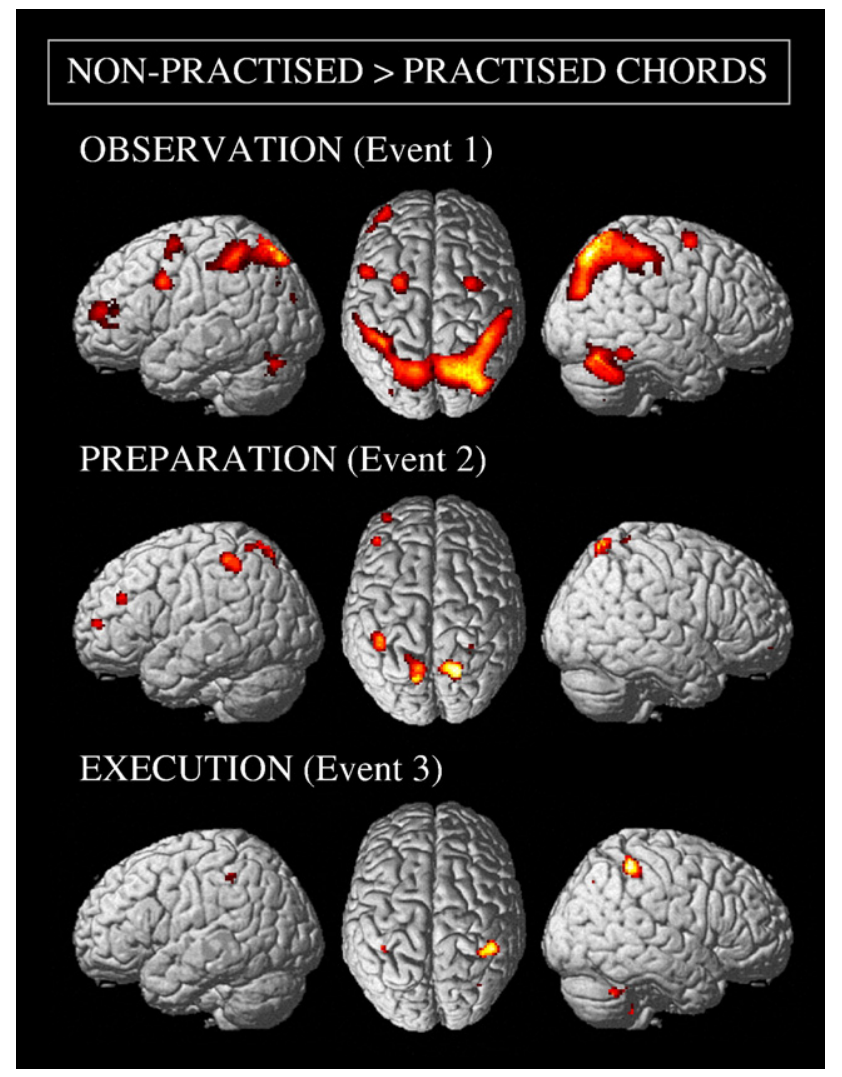

Fig. 4. Direct comparisons between non-practised and practised chord conditions for observation, motor preparation and execution events.

\section{Prefrontal activations}

Three prefrontal regions were found activated in the present study (Table 3). In the left hemisphere, a rostral and a caudal focus were found in the middle frontal gyrus (MFG), whereas in the right hemisphere, only the caudal part of the MFG was activated. Each of these regions exhibited a characteristic pattern of modulation by event, chord type and group (Fig. 3). These patterns were quantified by extracting the percent BOLD signal change for each condition at the local maxima within these regions (Fig. 5). The related, fourfactorial ANOVA indicated a significant overall effect of chord type $(F=10.95, p<0.01)$, which was modulated by event, by area and by the combination of event, area and group. Second, the interaction between event and area was highly significant $(F=24.75, p<0.001)$. In the left MFG, robust positive activations were only present in Events 1 and 2, with a significant increase between these events in the left rostral MFG only. In contrast, the right caudal MFG was only marginally activated during Event 1 but showed sustained activity during Events 2 and 3 (see Figs. 3 and 5).

Simple effect analyses of the percent BOLD signal change, run separately for each combination of event and area, indicated that the effects of chord type were largely restricted to the left rostral MFG (Events 1 and 2), with only marginal or non-significant effects for the two caudal sites. This is backed-up by the contrast maps for non-practised vs. practised chords (Fig. 4), where the left rostral MFG showed a strong differential activation in Event 1, with smaller foci in the two left prefrontal sites in Event 2.

Finally, during action observation the non-guitarists tended to show stronger BOLD signal intensities than the guitarists in all three prefrontal areas. This trend reversed during motor preparation, as confirmed by a significant interaction between event (1 vs. 2 ) and group $(F=8.57, p<0.01)$.

Table 2

Direct contrasts between non-practised and practised chord conditions: MNI coordinates of local maxima of activated areas as shown in Fig. 4 [ATB: most probable anatomical region in the Anatomy Toolbox 1.4, Eickhoff et al., 2005; asterisks $\left(^{*}\right)$ denote assigned areas]

\begin{tabular}{|c|c|c|c|c|c|c|c|c|c|c|}
\hline \multirow[t]{2}{*}{ Anatomical region } & \multicolumn{5}{|l|}{ Left } & \multicolumn{5}{|c|}{ Right } \\
\hline & $x$ & $y$ & $z$ & $Z$-score & ATB & $x$ & $y$ & $z$ & $Z$-score & ATB \\
\hline \multicolumn{11}{|l|}{ Event 1} \\
\hline Superior parietal lobule & -12 & -72 & 54 & 4.73 & & 24 & -70 & 58 & 5.50 & \\
\hline Precuneus & -14 & -68 & 62 & 4.71 & & 8 & -70 & 62 & 5.44 & \\
\hline Inferior parietal lobule & -36 & -44 & 46 & 4.06 & $30 \%$ hiP2 & 54 & -32 & 56 & 4.32 & \\
\hline Inferior temporal gyrus & & & & & & 52 & -46 & -16 & 4.67 & \\
\hline Superior frontal sulcus & -26 & 2 & 52 & 4.25 & $10 \%$ BA 6 & 30 & 0 & 66 & 3.93 & $10 \%$ BA6 \\
\hline Precentral gyrus (PMv) & -48 & 8 & 36 & 4.16 & $30 \%$ BA 44 & & & & & \\
\hline Middle frontal gyrus (rostral) & -38 & 56 & 14 & 4.24 & & & & & & \\
\hline Cerebellum (Crus I) & -30 & -60 & -36 & 4.45 & & 40 & -64 & -26 & 4.73 & \\
\hline Cerebellum (Crus II) & -10 & -78 & -38 & 4.56 & & & & & & \\
\hline \multicolumn{11}{|l|}{ Event 2} \\
\hline Precuneus & -14 & -60 & 64 & 3.78 & & 14 & -62 & 62 & 4.19 & \\
\hline Inferior parietal lobule / postcentral gyrus & -42 & -42 & 54 & 3.67 & $50 \% \mathrm{BA} 2 *$ & 28 & -46 & 70 & 3.16 & $50 \%$ BA $2 *$ \\
\hline Middle frontal gyrus (caudal) & -44 & 38 & 28 & 3.73 & & & & & & \\
\hline Middle frontal gyrus (rostral) & -34 & 54 & 10 & 3.45 & & & & & & \\
\hline Cerebellum (VII) & -20 & -76 & -50 & 3.27 & & & & & & \\
\hline \multicolumn{11}{|l|}{ Event 3} \\
\hline Inferior parietal lobule & -38 & -42 & 48 & 3.36 & $30 \%$ BA2 & 44 & -42 & 56 & 3.96 & \\
\hline Cerebellum (Crus I) & & & & & & 38 & -56 & -34 & 3.40 & \\
\hline Cerebellum (VIII) & -24 & -64 & -42 & 3.31 & & 24 & -42 & -48 & 3.35 & \\
\hline
\end{tabular}




\section{Discussion}

Three main findings were obtained in this study and will be discussed in turn: First, the results for the imitation of hand actions across practice conditions replicate and extend previous findings in several ways. Second, the pattern of activations in the DLPFC confirms the hypothesis that in imitation learning the left rostral MFG engages in operations of selection and combination of motor representations in the MNS (Buccino et al., 2004a). In addition, our data support the proposal by Shallice (2004) that the right MFG is primarily involved in monitoring if a newly configured motor plan is implemented and executed in accordance with task goals. Third, the MNS was more strongly involved during the observation of novel actions than of previously practised actions. This finding indicates a strong role of the MNS in imitation learning, and thus complements the work by Calvo-Merino et al. $(2005,2006)$ and Cross et al. (2006) which suggested a primary role of the MNS in representing previously acquired motor skills.

\section{Imitation of hand actions}

In order to identify regions with mirror properties, we computed a conjunction between action observation, motor preparation and execution. This indicated a parieto-premotor network (inferior and superior parietal lobules as well as ventral and dorsal premotor regions) similar to that reported in previous studies (e.g., Grèzes et al., 2003). The interpretation of the roles of inferior parietal lobule and ventral premotor cortex (PMv) as core components of the mirror neuron system is straightforward (Buccino et al., 2004a; Rizzolatti and Craighero, 2004). Both areas most likely code the identity and posture of individual elements in the display as potential own actions (e.g., an extended index finger and a flexed ring finger). During observation, the fingers on the screen are mapped onto the observer's own motor repertoire, and these elementary representations remain activated through to execution. In their recent meta-analysis of related neuroimaging studies, Chouinard and Paus (2006) report a focus in left PMv as conjunct between studies on the observation and studies on the execution of object-related actions $(-58,6,28$ in Talairach coordinates; approximately $-59,5,31$ in MNI coordinates) which closely matches that in the present study. Furthermore, it is reassuring that the direct contrasts between events (bottom panel of Fig. 2) indicate little change in the regions of the MNS, whereas the SMA, primary sensory-motor areas and areas involved in complex tactile exploration exhibit dramatic changes.

The bilateral activation in superior frontal gyrus can be interpreted in several ways: According to Buccino et al. (2004a), it reflects the involvement of dorsal premotor cortex (PMd) in preparing the proprioceptive and motor execution aspects of the required action. Further, the cluster in the right hemisphere is coextensive with the focus for rostral PMd in the meta-analysis by Chouinard and Paus (2006), where this area (TAL: 28, 0, 52; MNI: $28,-3,56)$ was consistently involved in the selection of motor responses based on spatial or arbitrary cues. Our left cluster, however, is located more medially and dorsally than their focus for left rostral PMd (TAL: $-32,-2,50$; MNI: $-32,-5,54)$. A second possibility is that the superior frontal activation reflects oculomotor processing, given its proximity to the location of the human frontal eye field (FEF) according to Grosbras et al. (2005) (TAL: $-30,-8$, 50 and 36, -8, 48; MNI: $-30,-11,54$ and 36, -11, 52). Both interpretations, PMd and FEF, are not mutually exclusive and reflect the requirement of the chord task to code the location of each effector on the fretboard, that is, for spatially oriented behaviour.

Taken together, the activations in PMv and PMd likely reflect a parallel specification of imitative action, with PMv specifying individual effectors and their posture as relayed via the inferior parietal lobule, and PMd specifying spatial positions for each effector, likely relayed via the superior parietal lobule and hIP2 (with the latter as a possible, architectonically defined correlate of functionally defined area AIP, see Choi et al., 2006; Grefkes et al., 2002; Grefkes and Fink, 2005).

Two points are noteworthy regarding the observed eventspecific activations, firstly the extension of the ventral premotor

Table 3

MNI coordinates of local maxima in the middle frontal gyrus (MFG), given separately for each event, non-guitarists (NG) and guitarists (G) and for non-practised (np) and practised (pr) chords

\begin{tabular}{|c|c|c|c|c|c|c|c|c|c|c|c|c|}
\hline & \multicolumn{4}{|c|}{ Left rostral MFG } & \multicolumn{4}{|c|}{ Left caudal MFG } & \multicolumn{4}{|c|}{ Right caudal MFG } \\
\hline & $x$ & $y$ & $z$ & $Z$-score & $x$ & $y$ & $z$ & $Z$-score & $x$ & $y$ & $z$ & $Z$-score \\
\hline \multicolumn{13}{|l|}{ Event 1} \\
\hline NG/np & -40 & 52 & 4 & 4.64 & -46 & 38 & 30 & 4.54 & 44 & 44 & 26 & 4.03 \\
\hline $\mathrm{NG} / \mathrm{pr}$ & & & & & -48 & 32 & 34 & 4.24 & & & & \\
\hline G/np & -38 & 56 & 16 & 3.24 & -48 & 34 & 32 & 4.29 & 52 & 34 & 30 & 3.21 \\
\hline \multicolumn{13}{|l|}{$\mathrm{G} / \mathrm{pr}$} \\
\hline \multicolumn{13}{|l|}{ Event 2} \\
\hline NG/np & -34 & 52 & 10 & 3.91 & -40 & 36 & 26 & 4.20 & 34 & 40 & 30 & 4.20 \\
\hline $\mathrm{NG} / \mathrm{pr}$ & -34 & 52 & 12 & 3.31 & -34 & 36 & 30 & 4.06 & 34 & 38 & 32 & 4.28 \\
\hline G/np & -30 & 52 & 16 & 5.23 & -34 & 36 & 30 & 5.07 & 34 & 40 & 26 & 5.91 \\
\hline $\mathrm{G} / \mathrm{pr}$ & -34 & 52 & 18 & 3.40 & -36 & 38 & 30 & 3.79 & 34 & 44 & 26 & 4.17 \\
\hline \multicolumn{13}{|l|}{ Event 3} \\
\hline NG/np & & & & & & & & & 36 & 44 & 30 & 3.36 \\
\hline NG/pr & & & & & -30 & 36 & 26 & 3.59 & 36 & 42 & 28 & 3.81 \\
\hline $\mathrm{G} / \mathrm{np}$ & & & & & -38 & 38 & 26 & 4.13 & 40 & 44 & 24 & 4.64 \\
\hline $\mathrm{G} / \mathrm{pr}$ & & & & & -36 & 40 & 26 & 4.07 & 40 & 46 & 18 & 4.23 \\
\hline
\end{tabular}


OBSERVATION: left rostral MFG

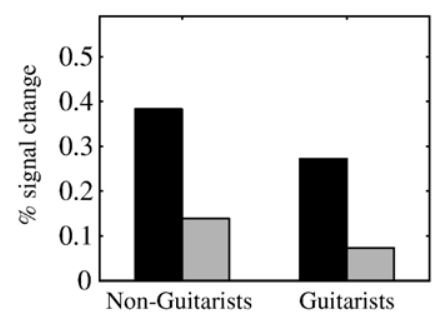

PREPARATION

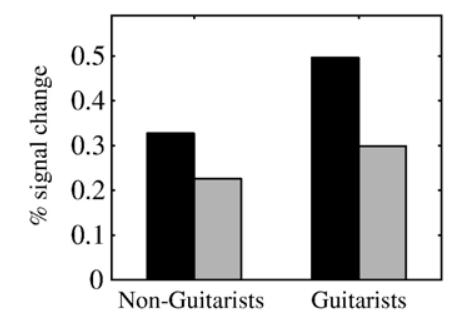

EXECUTION

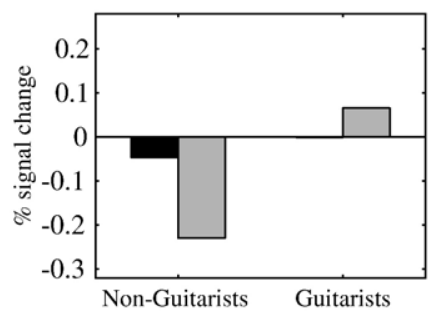

left caudal MFG
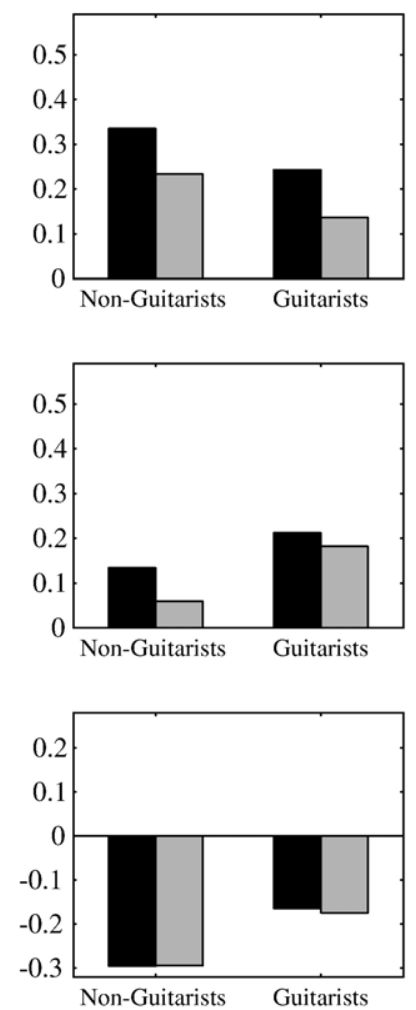

right caudal MFG
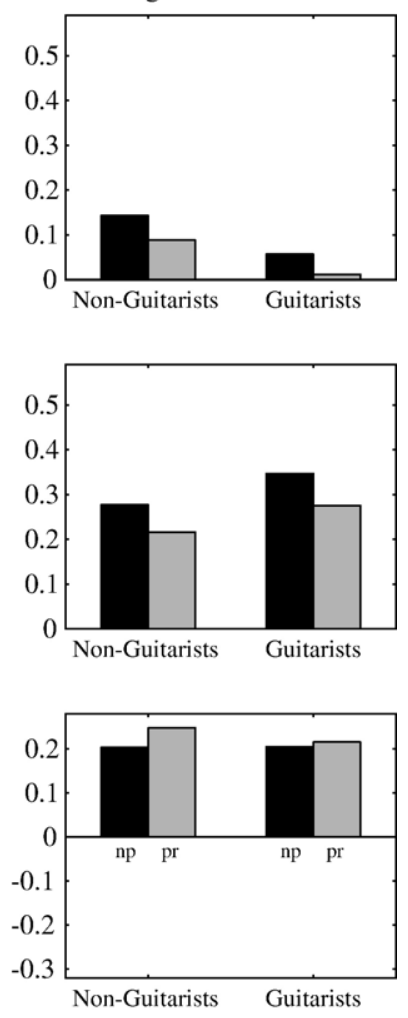

Fig. 5. Percent BOLD signal change for the effects of event, group and chord type at the central voxels of three prefrontal regions. Left column: left rostral MFG $(-38,58,10)$; center column: left caudal MFG $(-44,36,32)$; right column: right caudal MFG $(36,42,28)$. Coordinates are the local maxima in the conjunction between events with significant activation of each region, across all participants and both chord types. Black bars show parameter estimates for non-practised chords, and grey bars those for practised chords.

focus into the pars opercularis of inferior frontal gyrus (dPO and vPO) during Events 2 and 3 and secondly the activation of primary sensory-motor areas during the preparatory Event 2. In a reanalysis of studies on the imitation of simple finger movements, Molnar-Szakacs et al. (2005) found dPO involved in imitation and, less strongly, in observation, whereas vPO was exclusively activated during imitation. Based on this dissociation, the authors proposed a functional segregation within $\mathrm{PO}$, where $\mathrm{dPO}$ is a classical mirror neuron area, whereas vPO provides a forward model of the expected sensory consequences of the action. Our data qualify this hypothesis in several ways. First, we showed that the ventral premotor cortex also exhibits mirror properties, as PMv was found activated in the conjunction analysis as well as in the underlying basic contrasts. These clusters only slightly invaded the adjacent dPO. As noted above, this conclusion is also supported by the meta-analysis of Chouinard and Paus (2006). Second, the focus in PMv increasingly extended into $\mathrm{dPO}$ and $\mathrm{vPO}$ during Events 2 and 3 , so that vPO was consistently activated during Event 3 (cf. Fig. 3). As proposed by Molnar-Szakacs et al. (2005), it appears possible that this event-specific activation reflects forward modeling in a network including posterior parietal and likely also cerebellar regions. While one would expect forward modeling to be primarily associated with motor execution, we found vPO already involved when the guitarists observed the non-practised chords (G/np-1). Indeed, forward modeling in advance of execution would be most useful when selecting amongst familiar but not recently practised actions.
The activation focus located in the sensorimotor cortex (M1, plus BA2 and OP1) during Event 2 seems to indicate a stronger 'motor readiness' of participants than in our first study and is consistent with the previously reported involvement of M1 in motor preparation (Endo et al., 1999; Ikeda et al., 1996; Kawashima et al., 1994) and motor imagery (Lotze et al., 1999; Porro et al., 2000; Schnitzler et al., 1997).

\section{Effects of practice and expertise on prefrontal activations}

A major aim of the present study was to assess the effects of practice on prefrontal activations in imitation learning. To this end, participants had practised four chords in a single session one day before the scanning, which showed clear practice effects in the behavioural testing. This most likely reflected the fact that the practised chords were recognised and executed as complete units, whereas participants were still engaged in configuring the nonpractised chords (Hazeltine et al., in press). According to our model of imitation learning (Buccino et al., 2004a), the selection and combination of motor elements into novel actions proceeds under the control of the DLPFC. The present data support this hypothesis in the following ways. First, during action observation the left rostral MFG (most likely area 46) was more strongly involved than in the previous study by Buccino et al. (2004a) (Fig. 3, top left panel), possibly as a result of the additional training session and of the related greater 'motor readiness' of the participants in our current study. This demonstration of prefrontal involvement during action 
observation is an important extension of our previous results, where activations in the left rostral MFG were largely restricted to the subsequent motor preparatory event, with the inherent ambiguity of interpretation of prefrontal activations in the hold period of delayed response tasks (Passingham, 1993). Second, area 46 was more strongly activated during action observation and motor preparation of the non-practised chords compared to the practised chords, with the latter exhibiting reduced or absent activations. Third, reliable effects of chord type were observed on the BOLD signal specifically for the left rostral MFG. These findings render an explanation in terms of passive maintenance of sensory or motor representations unlikely and support an interpretation of DLPFC function in terms of integrating this information and selecting appropriate behaviour (Passingham and Sakai, 2004). Finally, during motor execution, the left prefrontal foci showed no or even reduced BOLD signals relative to baseline, whereas the right caudal MFG showed sustained signal amplitudes in Events 2 and 3. These differential effects are well in line with Shallice's (2004) proposal of a left DLPFC dominance in modulating lower level systems (here: the MNS), whereas the right DLPFC is dominant in monitoring operations (here: checking whether motor preparation and execution match the observed model).

How are these findings to be interpreted in the context of previous work on prefrontal involvement in motor learning? In general, the role of the prefrontal cortex in dealing with non-routine operations is undisputed (Miller and Cohen, 2001). Furthermore, it has been shown in a variety of cognitive and motor tasks that prefrontal activations are most pronounced for novel material and substantially reduce with practice, often to baseline levels (e.g., Petersen et al.'s, 1998, "scaffolding-storage" framework). In studies of non-imitative motor learning, such a redistribution of activations (Kelly and Garavan, 2005) has been demonstrated for sequencing tasks (Keele et al., 2003), bimanual coordination (Debaere et al., 2004) and force field adaptation (Shadmehr and Holcomb, 1997). However, rather indirect task instructions were typically used in these paradigms (e.g., trial-and-error learning of sequences of keypresses in Jenkins et al., 1994), and prefrontal activations might therefore primarily reflect the cognitive operations of understanding the required task, rather than top-down control of performance per se. In contrast, given the high degree of visuomotor compatibility in imitation paradigms (Meltzoff and Prinz, 2002), prefrontal engagement might not be expected. Our demonstration of transient left prefrontal involvement in imitation learning thus indicates that even in tasks allowing for a direct matching of visual and motor representations, top-down control of these elementary representations is present when learning novel material. Regarding the role of different prefrontal areas, further research is required to indicate whether the DLPFC is more generally associated with imitation tasks (Iacoboni, 2005), or whether our findings merely reflect the specific requirement of configurational tasks to manipulate and monitor several pieces of information that mid-dorsal prefrontal regions have been associated with (Petrides, 1995). To examine further our interpretation of the right DLPFC activations as reflecting monitoring operations (Shallice, 2004), future research should aim to replicate the present pattern of results independent of the effector used (left hand in our study).

As described above, expert guitarists showed significantly smaller BOLD signals in prefrontal regions during action observation and larger signals during motor preparation. As one would expect, the guitarists most likely tended to exert less supervisory control during observation, given that both chord types were familiar to them, and they exerted more control during motor preparation, which was reflected in the higher scores for imitation quality. However, compared to the robust practice effects, the group differences were rather small. In particular, larger group differences might have been expected specifically for the non-practised chords, which were familiar to the guitarists only. Interestingly, however, practice and familiarity with the chords were indeed reflected in the hippocampal activations during Event 1, which were present in all conditions except when the non-guitarists observed the nonpractised chords. Nevertheless, also in the quality of imitations, both groups showed equivalent differences between the two chord types, and in the speed test after the scanning, the guitarists showed even stronger performance decrements for the non-practised chords than the non-guitarists. A viable explanation for this pattern of results is that the guitarists had engaged strongly in the practice phase and that, as the non-guitarists, they re-engaged strongly when confronted with the non-practised chords. Also, given that the guitarists were mainly trained in classical guitar, their spectrum of hand postures was extremely wide, and thus familiarity with the basic chords used in the present study was possibly less advantageous as for, e.g., folk guitarists with a typically more limited chord repertoire. Finally, the unusual arm and hand posture in which the chords were to be performed may have prevented the guitarists from fully benefitting from their expertise.

Effects of practice and expertise on the mirror neuron system and related areas

As predicted, activations in the MNS were stronger for nonpractised than for practised chords, in particular during action observation when all posterior parietal and premotor components of the MNS exhibited this effect. During the motor preparation and execution events, the posterior parietal areas continued to show differential activations between chord types.

The stronger involvement of the MNS for novel hand actions apparently contradicts the results of a number of previous studies. First, Buccino et al. (2004b) demonstrated that only actions that were present in the observer's motor repertoire produced activations in the MNS. More recently, Calvo-Merino et al. $(2005,2006)$ and Cross et al. (2006) showed stronger activations in the MNS when dancers observed dancing movements that were part of the individual's motor repertoire, compared to observation of matched, but nonpractised movements. How can these conflicting results be explained? Whereas participants in our study imitated the chords after observation, participants in Buccino et al.'s (2004b) study simply watched the different actions, participants in Calvo-Merino et al.'s $(2005,2006)$ studies were asked to judge 'how tiring' each movement was, and those in the study by Cross et al. (2006) judged how well they could dance each movement. It is plausible that the different outcomes between these studies and ours reflect the different aims of the observation (see also Grèzes et al., 1998). During observation not followed by imitation, observers tend to 'resonate' more strongly with actions already embodied in their own motor repertoire. In contrast, during observation in order to imitate, novel actions tend to induce stronger activations in the MNS than familiar actions. This likely results from a stronger modulatory input from prefrontal areas, which were not found activated in the above action observation studies. Accordingly, one should expect that a pure chord observation condition reduces activations in the MNS relative to an imitation condition. This was indeed demonstrated by Buccino et al. (2004a) for novel chords. Furthermore, we would 
predict that, when dancers were asked to actually imitate movements after the scanning, their MNS (and DLPFC) would be more strongly involved when observing non-familiar movements than familiar ones.

Whereas the moderate practice given in the present study indeed reduced prefrontal activations, it did not produce clear-cut shifts of activations to other cortical regions, in the sense of a genuine reorganisation (Kelly and Garavan, 2005). Although stronger activations for practised vs. non-practised chords were observed in a number of cortical areas, none of these differential activations corresponded to positive activations in the basic contrasts. Therefore, these findings must be treated with caution. A minimalist explanation would be that both elementary finger postures and learned configurations of these postures (i.e., complete chords) are represented in the MNS. Hence, large-scale shifts of activations to areas other than the MNS would not be expected as a result of practice. A likely exception might be an additional coding of highly practised actions in superior temporal regions (e.g., Calvo-Merino et al., 2005), which presumably requires longer practice periods than employed in the present study. Nevertheless, the stronger activation for practised chords in the left angular gyrus and the right posterior middle temporal gyrus in our study might be an early indication of such an experience-dependent plasticity of higher order visual areas.

Finally, we also observed some expertise effects in areas other than the prefrontal cortex. During action observation, the guitarists showed stronger activations in the right precentral gyrus, presumably reflecting their increased level of motor readiness, while the non-guitarists showed stronger activations in the right superior parietal lobule, presumably reflecting their emphasis on abstract visuospatial and/or kinesthetic analysis. During motor preparation and execution, a different sector of the precentral gyrus was more strongly activated in the guitarists, as well as the left inferior parietal lobule. The latter likely reflected a more elaborate representation of the chord postures in this mirror neuron area. During motor execution, the guitarists also exhibited a stronger activation in the parietal operculum ('OP 4', Eickhoff et al., 2006a,b), likely reflecting a more elaborate analysis of multimodal somatosensory input. Similarly as the group differences in prefrontal regions, also the above group effects were less pronounced than the practice effects in the present study; nevertheless, they fit well into the present explanatory framework.

In conclusion, we have demonstrated that the human MNS can be involved in the early stages of imitation learning. This finding is at variance with earlier views that suggested that this involvement requires motor experience with the observed action. When subsequent imitation is required, observed unfamiliar actions are decomposed into familiar elements via motor resonance in the MNS (Buccino et al., 2004a). The combination of these elements into a novel configural action most likely proceeds under the supervisory control of the left dorsolateral prefrontal cortex.

\section{Acknowledgments}

We would like to thank B. Elghahwagi, G. Oefler, J. Kirchhoff and W. Rieger (Jülich) for their kind support while conducting the study. Special thanks to H.-W. Huppertz, docent at the Musikhochschule Aachen, and his students for their enthusiasm and participation in the study, to M. Ziessler (Liverpool) for helpful discussions and to K. Maier (Lancaster) for her indispensable help with participant recruitment and training, and with scoring the videorecordings. T. Shallice (London and Trieste) and an anonymous referee provided helpful comments on an earlier draft of this paper. This work was supported by grants from the British Academy (SG-36132) and the Leverhulme Trust (F/00 185/K) to S. Vogt. G. Rizzolatti and G. Buccino were supported by ASI (Agenzia Spaziale Italiana) and MIUR (Ministero dell'Università e della Ricerca).

\section{References}

Bird, G., Heyes, C., 2005. Effector-dependent learning by observation of a finger movement sequence. J. Exp. Psychol., Hum. Percept. Perform. 31, 262-275.

Borroni, P., Montagna, M., Cerri, G., Baldissera, F., 2005. Cyclic time course of motor excitability modulation during the observation of a cyclic hand movement. Brain Res. 1065, 115-124.

Binkofski, F., Buccino, G., Posse, S., Seitz, R.J., Rizzolatti, G., Freund, H.J., 1999. A fronto-parietal circuit for object manipulation in man: evidence from an fMRI-study. Eur. J. Neurosci. 11, 3276-3286.

Buccino, G., Vogt, S., Ritzl, A., Fink, G.R., Zilles, K., Freund, H.-J., Rizzolatti, G., 2004a. Neural circuits underlying imitation learning of hand actions: an event-related fMRI study. Neuron 42, 323-334.

Buccino, G., Lui, F., Canessa, N., Patteri, I., Langravinese, G., Benuzzi, F., et al., 2004b. Neural circuits involved in the recognition of actions performed by nonconspecifics: an fMRI study. J. Cogn. Neurosci. 16, 114-126.

Byrne, R., 2003. Imitation as behaviour parsing. Philos. Trans. R. Soc. London, Ser. B 358, 529-536.

Calvo-Merino, B., Glaser, D.E., Grèzes, J., Passingham, R.E., Haggard, P., 2005. Action observation and acquired motor skills: an fMRI study with expert dancers. Cereb. Cortex 15, 1243-1249.

Calvo-Merino, B., Grèzes, J., Glaser, D.E., Passingham, R.E., Haggard, P., 2006. Seeing or doing? Influence of visual and motor familiarity in action observation. Curr. Biol. 16, 1905-1910.

Choi, H.-J., Zilles, K., Mohlberg, H., Schleicher, A., Fink, G.F., Armstrong, E., Amunts, K., 2006. Cytoarchitectonic identification and probabilistic mapping of two distinct areas within the anterior ventral bank of the human intraparietal sulcus. J. Comp. Neurol. 495, 53-69.

Chouinard, P.A., Paus, T., 2006. The primary motor and premotor areas of the human cerebral cortex. Neuroscientist 12, 1-10.

Cross, E.S., Hamilton, A.F., Grafton, S.T., 2006. Building a motor simulation de novo: observation of dance by dancers. NeuroImage 31 , 1257-1267.

Debaere, F., Wenderoth, N., Sunaert, S., Van Hecke, P., Swinnen, S.P., 2004. Changes in brain activation during the acquisition of a new bimanual coordination task. Neuropsychologia 42, 855-867.

Eickhoff, S.B., Stephan, K.E., Mohlberg, H., Grefkes, C., Fink, G.R., Amunts, K., Zilles, K., 2005. A new SPM toolbox for combining probabilistic cytoarchitectonic maps and functional imaging data. NeuroImage 25, 1325-1335.

Eickhoff, S.B., Schleicher, A., Zilles, K., Amunts, K., 2006a. The human parietal operculum. I. Cytoarchitectonic mapping of subdivisions. Cereb. Cortex 16, 254-267.

Eickhoff, S.B., Amunts, K., Mohlberg, H., Zilles, K., 2006b. The human parietal operculum II. Stereotaxic maps and correlation with functional imaging results. Cereb. Cortex 16, 268-279.

Endo, H., Kizuka, T., Masuda, T., Takeda, T., 1999. Automatic activation in the human primary motor cortex synchronized with movement preparation. Cogn. Brain Res. 3, 229-239.

Friston, K.J., Holmes, A.P., Worsley, K.J., 1999. How many subjects constitute a study? NeuroImage 10, 1-5.

Grefkes, C., Fink, G.R., 2005. The functional organization of the intraparietal sulcus in humans and monkeys. J. Anat. 207, 3-17.

Grefkes, C., Weiss, P.H., Zilles, K., Fink, G.R., 2002. Crossmodal processing of object features in human anterior intraparietal cortex: an 
fMRI study implies equivalencies between humans and monkeys. Neuron 35, 173-184.

Grèzes, J., Costes, N., Decety, J., 1998. Top-down effect of strategy on the perception of human biological motion: a PET investigation. Cogn. Neuropsychol. 15, 553-582.

Grèzes, J., Armony, J.L., Rowe, J., Passingham, R.E., 2003. Activations related to "mirror" and "canonical" neurones in the human brain: an fMRI study. NeuroImage 18, 928-937.

Grosbras, M.-H., Laird, A.R., Paus, T., 2005. Cortical regions involved in eye movements, shifts of attention, and gaze perception. Hum. Brain Mapp. 25, 140-154.

Hazeltine, E., Aparicio, P., Weinstein, A., Ivry, R., in press. Configural response learning: the acquisition of a nonpredictive motor skill. Journal of Experimental Psychology: Human Perception and Performance.

Iacoboni, M., 2005. Neural mechanisms of imitation. Curr. Opin. Neurobiol. $15,632-637$.

Iacoboni, M., Woods, R.P., Brass, M., Bekkering, H., Mazziotta, J.C., Rizzolatti, G., 1999. Cortical mechanisms of human imitation. Science 286, 2526-2528.

Ikeda, A., Lüders, H.O., Collura, T.F., Burgess, R.C., Morris, H.H., Hamano, T., Shibasaki, H., 1996. Subdural potentials at orbitofrontal and mesial prefrontal areas accompanying anticipation and decision making in humans: a comparison with Bereitschaftspotential. Electroencephalogr. Clin. Neurophysiol. 98, 206-212.

Jeannerod, M., 1994. The representing brain: neural correlates of motor intention and imagery. Behav. Brain Sci. 17, 187-202.

Jenkins, I.H., Brooks, D.J., Nixon, P.D., Frackowiak, R.S.J., Passingham, R.E., 1994. Motor sequence learning: a study with positron emission tomography. J. Neurosci. 14, 3775-3790.

Kawashima, R., Roland, P., O'Sullivan, B.T., 1994. Fields in human motor areas involved in preparation for reaching, actual reaching, and visuomotor learning: a positron emission tomography study. J. Neurosci. $14,3462-3474$

Keele, S.W., Ivry, R., Mayr, U., Hazeltine, E., Heuer, H., 2003. The cognitive and neural architecture of sequence representation. Psychol. Rev. 110, 316-339.

Kelly, A.M.C., Garavan, H., 2005. Human functional neuroimaging of brain changes associated with practice. Cereb. Cortex 15, 1089-1102.

Koski, L., Iacoboni, M., Dubeau, M.-C., Woods, R.P., Mazziotta, J.C., 2003. Modulation of cortical activity during different imitative behaviors. J. Neurophysiol. 89, 460-471.

Lotze, M., Montoya, P., Erb, M., Hülsmann, E., Flor, H., Klose, U., Birbaumer, N., Grodd, W., 1999. Activation of cortical and cerebellar motor areas during executed and imagined hand movements: an fMRI study. J. Cogn. Neurosci. 11, 491-501.

Mattar, A.G., Gribble, P.L., 2005. Motor learning by observation. Neuron 46, 153-160.

Miller, E.K., Cohen, J.D., 2001. An integrative theory of prefrontal cortex function. Annu. Rev. Neurosci. 24, 167-202.

Meltzoff, A.N., Prinz, W. (Eds.), 2002. The Imitative Mind: Development, Evolution, and Brain Bases. Cambridge Univ. Press, Cambridge.
Molnar-Szakacs, I., Iacoboni, M., Koski, L., Mazziotta, J.C., 2005. Functional segregation within pars opercularis of the inferior frontal gyrus: evidence from fMRI studies of imitation and action observation. Cereb. Cortex 15, 986-994.

Passingham, R.E., 1993. The Frontal Lobes and Voluntary Action. Oxford Univ. Press, Oxford.

Passingham, D., Sakai, K., 2004. The prefrontal cortex and working memory: physiology and brain imaging. Curr. Opin. Neurobiol. 14, 163-168.

Petersen, S.E., van Mier, H., Fiez, J.A., Raichle, M.E., 1998. The effects of practice on the functional anatomy of task performance. Proc. Natl. Acad. Sci. U. S. A. 95, 853-860.

Petrides, M., 1995. Functional organization of the human frontal cortex for mnemonic processing: evidence from neuroimaging studies. In: Grafman, J., Holyoak, K.J., Boller, F. (Eds.), Structure and Functions of the Human Prefrontal Cortex. Annals of the New York Academy of Sciences, vol. 769, pp. 85-96.

Porro, C.A., Cettolo, V., Francescato, M.P., Baraldi, P., 2000. Ipsilateral involvement of primary motor cortex during motor imagery. Eur. J. Neurosci. 12, 3059-3063.

Rizzolatti, G., 2005a. The mirror neuron system and its function in imitation. Anat. Embryol. 210, 419-421.

Rizzolatti, G., 2005b. The mirror neuron system and imitation. In: Hurley, S., Chater, N. (Eds.), Perspectives on Imitation: From Neuroscience to Social Science, vol. 1. MIT Press, Cambridge, MA, pp. 55-76.

Rizzolatti, G., Craighero, L., 2004. The mirror-neuron system. Annu. Rev. Neurosci. 27, 169-192.

Rizzolatti, G., Fogassi, L., Gallese, V., 2001. Neurophysiological mechanisms underlying the understanding and imitation of action. Nat. Rev., Neurosci. 2, 661-670.

Rizzolatti, G., Fadiga, L., Fogassi, L., Gallese, V., 2002. From mirror neurons to imitation: facts and speculations. In: Meltzoff, A.N., Prinz, W. (Eds.), The Imitative Mind: Development, Evolution, and Brain Bases. Cambridge Univ. Press, Cambridge, pp. 247-266.

Schnitzler, A., Salenius, S., Salmelin, R., Jousmäki, V., Hari, R., 1997. Involvement of primary motor cortex in motor imagery: a neuromagnetic study. NeuroImage 6, 201-208.

Shadmehr, R., Holcomb, H.H., 1997. Neural correlates of motor memory. Science 277, 821-825.

Shallice, T., 2004. The fractionation of supervisory control, In: Gazzaniga, M.S. (Ed.), The Cognitive Neurosciences, Third edition. MIT Press, Cambridge, MA, pp. 943-956.

Stoeckel, M.C., Weder, B., Binkofski, F., Buccino, G., Shah, N.J., Seitz, R.J., 2003. A fronto-parietal circuit for tactile object discrimination: an event-related fMRI study. NeuroImage 19, 1103-1114.

Vogt, S., 1995. On relations between perceiving, imagining and performing in the learning of cyclical movement sequences. Br. J. Psychol. 86, 191-216

Vogt, S., Thomaschke, R., 2007. From visuo-motor interactions to imitation learning: behavioural and brain imaging studies. J. Sports Sci. 25, 497-517.

Wilson, M., Knoblich, G., 2005. The case for motor involvement in perceiving conspecifics. Psychol. Bull. 131, 460-473. 